\title{
Photon scattering from strongly driven atomic ensembles
}

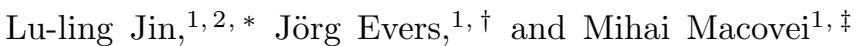 \\ ${ }^{1}$ Max-Planck Institut für Kernphysik, Saupfercheckweg 1, D-69117 Heidelberg, Germany \\ ${ }^{2}$ Department of Physics, Northwest University, Xi'an 710069, Shaanxi, China
}

(Dated: June 10, 2022)

\begin{abstract}
The second order correlation function for light emitted from a strongly and near-resonantly driven dilute cloud of atoms is discussed. Because of the strong driving, the fluorescence spectrum separates into distinct peaks, for which the spectral properties can be defined individually. It is shown that the second-order correlations for various combinations of photons from different spectral lines exhibit bunching together with super- or sub-Poissonian photon statistics, tunable by the choice of the detector positions. Additionally, a Cauchy-Schwarz inequality is violated for photons emitted from particular spectral bands. The emitted light intensity is proportional to the square of the number of particles, and thus can potentially be intense. Three different averaging procedures to model ensemble disorder are compared.
\end{abstract}

PACS numbers: $42.25 . \mathrm{Hz}, 42.50 . \mathrm{Hz}, 42.50 . \mathrm{Ar}, 42.50 . \mathrm{Dv}$

\section{INTRODUCTION}

The characterization of light is an ubiquitous problem, and a convenient formalism for this is the use of correlation functions. However it is well known that it is not possible to sufficiently distinguish the nature of a given light source from only the first-order correlation function [1]. In particular, the quantum properties of light cannot be extracted from the first order correlation function. This motivated the study of second-order correlations, initiated by the intensity-correlation experiments conducted by Hanbury-Brown and Twiss [2]. Subsequently, secondorder correlation measurements have found applications in many fields of modern physics 3 3uch as astronomy [4], optics 5], high-energy physics [6], condensed matter physics [7, 8] and atomic physics [9].

Next to the characterization of a physical system via higher-order correlations, it has also been suggested to use strongly correlated particles or even entangled particles as input to a system for various applications, with the most obvious examples of quantum computation, quantum communication and quantum information processing. This prompts the question for efficient sources of correlated photons. A standard method for generating entangled photons is the parametric down-conversion processes [10]. Alternatively, entangled photons can be generated in four-wave mixing [11, 12] or electromagnetically induced transparency [13] processes. Further, an experiment on photon anti-bunching in phase-matched multi-atom resonance fluorescence was reported in [14]. Non-classical photon pairs for scalable quantum communication with atomic ensembles and ultraviolet entangled photons in a semiconductor were generated in [15] and [16], respectively. An atomic memory for correlated pho-

\footnotetext{
*Electronic address: jinll@nwu.edu.cn

${ }^{\dagger}$ Electronic address: joerg.evers@mpi-hd.mpg.de

${ }^{\ddagger}$ Electronic address: mihai.macovei@mpi-hd.mpg.de
}

ton states was demonstrated in 17], and a heralded entanglement source was realized in [18]. However, despite the large variety of sources, in many cases applications are restricted by a limited production rate for correlated photons, and by the lack of sources with tunable correlations.

Interestingly, it has been shown that disordered media can sometimes exhibit surprising correlation or coherence effects. For example, speckle patterns in the quantum correlations within light scattered by a disordered medium were observed in [19]. A cooperative radiation force in the presence of disorder was observed as well [20]. An experimental investigation of the origin of disorder in parametrically excited waves on a fluid surface (Faraday waves) was performed in 21]. Instabilities of waves in nonlinear disordered media and subwavelength spatial

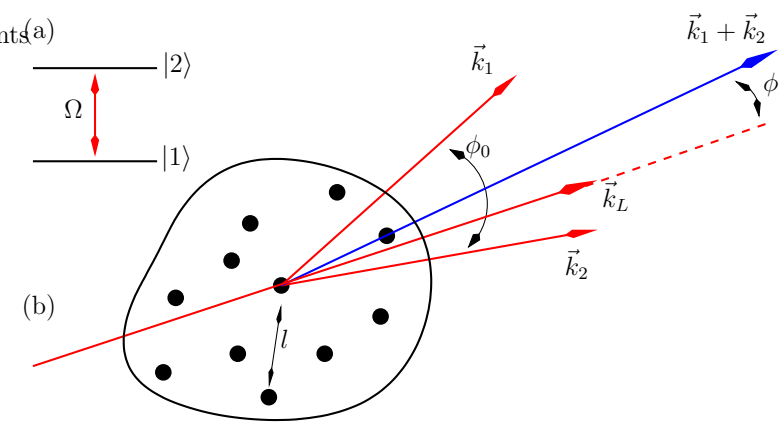

FIG. 1: (Color online) Schematic setup of a dilute atomic ensemble pumped with a coherent field with wave-vector $\vec{k}_{L}$. (a) shows the energy levels of each of the ensemble particles, and the interaction with the strong coherent light with coupling strength $\Omega$. (b) depicts the ensemble with typical interparticle distance length scale denoted by $l$ with $l \gg 2 \pi / k_{L}$. We consider the case of photon pair emission in forward direction and denote the angle between the two emitted photons with wave-vectors $\vec{k}_{1}$ and $\vec{k}_{2}$ as $\phi_{0}$, and $\vec{k}_{1}+\vec{k}_{2} \approx 2 \vec{k}_{L}$. The direction of the emission cone defined by $\vec{k}_{1}$ and $\vec{k}_{2}$ is characterized by the angle $\phi$ between $\vec{k}_{1}+\vec{k}_{2}$ and $\vec{k}_{L}$. 
correlations in near-field speckle patterns were discussed in 22, 23]. Correlation and coherence can also be observed in the context of localization [24]. Furthermore, coherent backscattering was investigated in [25], and random lasing in [26 28].

In this paper, we study the generation of correlated light from a disordered many-particle system, and in particular focus on the case of a strong near-resonant driving of the ensemble. Due to the strong driving, the fluorescence spectrum dissolves into distinct lines, which can be characterized separately. We then investigate secondorder correlation functions of light scattered into the various spectral lines. The disordered ensemble is modelled by calculating the emitted light for a fixed orientation between the atoms, followed by a suitable configuration averaging. For this, we compare three different averaging procedures. We find that the second-order correlation functions typically exhibit strong correlations if detected close to the forward scattering direction, whereas the corresponding first-order correlation functions are isotropic. In particular, bunching together with super- or sub-Poissonian photon statistics can be achieved for different combinations of spectral lines, and in some cases the statistics is tunable by the choice of the detector positions. We additionally demonstrate that light emitted from certain spectral bands violates a Cauchy-Schwarz inequality. Since the unnormalized second-order correlation functions of the emitted light scales with the number of particle in the ensemble squared, intense beams of correlated photons can be generated.

\section{THEORETICAL CONSIDERATIONS}

\section{A. The model}

We proceed by investigating an atomic sample of arbitrary shape and of characteristic size $d$, consisting of distinguishable non-overlapping two-level particles. We assume conditions such that multiple scattering effects can be neglected, i.e., we consider the single scattering regime in which the photon mean free path length is of the order of the sample size or larger. Related, we also restrict the typical inter-particle separation $l$ to satisfy

$$
\lambda_{L} \ll l \ll d
$$

with $d / c<\tau_{s}$, where $\tau_{s}$ is the spontaneous decay time. All particles have identical atomic transition frequencies $\omega_{0}$, and are localized at random positions $\vec{r}_{j}$ with $j \in\{1,2, \cdots, N\}$. We define the interparticle separation vectors as $\vec{r}_{i j}=\vec{r}_{i}-\vec{r}_{j}$. The external laser field has frequency $\omega_{L}=c k_{L}=2 \pi c / \lambda_{L}$, wave vector $\vec{k}_{L}$ and wavelength $\lambda_{L}$ (see Fig. 1).

Under the action of the laser field, the system is best described in a suitable dressed state picture. In electric dipole and rotating wave approximations, the system
Hamiltonian can be written as $H=H_{0}+H_{I}$, where 29]

$$
\begin{aligned}
& H_{0}=\sum_{k} \hbar\left(\omega_{k}-\omega_{L}\right) a_{k}^{\dagger} a_{k}+\sum_{j=1}^{N} \hbar \tilde{\Omega}_{j} R_{z j}, \\
& H_{I}=i \sum_{k} \sum_{j=1}^{N}\left(\vec{g}_{k} \cdot \vec{d}_{j}\right)\left\{a_{k}^{\dagger} S_{j}^{-} e^{-i\left(\vec{k}-\vec{k}_{L}\right) \cdot \vec{r}_{j}}-\text { H.c. }\right\} \\
& S_{j}^{-}=\frac{R_{z j}}{2} \sin 2 \theta_{j}-R_{21}^{(j)} \sin ^{2} \theta_{j}+R_{12}^{(j)} \cos ^{2} \theta_{j} .
\end{aligned}
$$

Here, $H_{0}$ represents the Hamiltonian of the free electromagnetic field (EMF) and free dressed atomic subsystems, respectively, while $H_{I}$ accounts for the interaction of the laser-dressed atoms with the EMF. $a_{k}$ and $a_{k}^{\dagger}$ are the field annihilation and creation operators obeying the standard commutation relations for bosons. The atomic operators $R_{\alpha \beta}^{(j)}=|\tilde{\alpha}\rangle_{j j}\langle\tilde{\beta}|$ describe the transitions between the dressed states $|\tilde{\beta}\rangle_{j}$ and $|\tilde{\alpha}\rangle_{j}$ in atom $j$ for $\alpha \neq \beta$ and dressed-state populations for $\alpha=\beta$, and satisfy the commutation relations of the $\mathrm{su}(2)$ algebra. The dressed states $|\tilde{\alpha}\rangle_{j}$ entering the operators $R_{\alpha \beta}^{(j)}$ can be represented through the bare states $|\alpha\rangle_{j}$ via the transformations

$$
\begin{aligned}
|1\rangle_{j} & =\sin \theta|\tilde{2}\rangle_{j}+\cos \theta|\tilde{1}\rangle_{j}, \\
|2\rangle_{j} & =\cos \theta|\tilde{2}\rangle_{j}-\sin \theta|\tilde{1}\rangle_{j} .
\end{aligned}
$$

We further defined

$$
R_{z j}=|\tilde{2}\rangle_{j j}\langle\tilde{2}|-| \tilde{1}\rangle_{j j}\langle\tilde{1}|,
$$

which is the difference of the upper and lower dressed state population. Further,

$$
\tilde{\Omega}=\tilde{\Omega}_{j}=\sqrt{\Omega^{2}+(\Delta / 2)^{2}}
$$

is the generalized Rabi frequency, with $2 \Omega=\left(\vec{d} \cdot \vec{E}_{L}\right) / \hbar$. Here, $\vec{E}_{L}$ is the electric laser field strength, and $\vec{d} \equiv \vec{d}_{j}$ is the transition dipole matrix element. The detuning $\Delta=\omega_{0}-\omega_{L}$ is characterized by $\cot 2 \theta=\Delta /(2 \Omega)$.

\section{B. Spectral decomposition}

In the following we make use of the fact that the resonance fluorescence spectrum of the light scattered by an atomic system pumped by a strong near-resonant driving field splits up into distinct lines, which are known as the Mollow spectrum [30]. The condition for well-resolved spectral lines is $\tilde{\Omega} \gg \gamma$, with $\gamma=1 / \tau_{s}$ being the singleatom spontaneous decay rate. In this limit of strong driving, it is reasonable to define optical properties for each of the spectral lines separately. Thus, it follows from the interaction Hamiltonian Eq. (2b) that the different operators in Eq. (2c) can be considered as sources of individual spectral lines. In particular, in the following we will denote light originating from $R_{z j} \sin (2 \theta) / 2$ as the central spectral component indicated by $C$, and $R_{21}^{(j)} \cos ^{2} \theta$ and 
$R_{12}^{(j)} \sin ^{2} \theta$ as the right $(\mathrm{R})$ and left $(\mathrm{L})$ spectral sideband components. These sidebands are emitted at frequencies $\omega_{+} \equiv \omega_{L}+2 \tilde{\Omega}$ and $\omega_{-} \equiv \omega_{L}-2 \tilde{\Omega}$, respectively 31,32 . In what follows, we shall use this decomposition to investigate the properties of the scattered light.

\section{First order correlations}

In order to calculate the light scattered by the cloud of two-level scatterers, we assume detection in the far-zone limit, that is, the linear dimension of the atomic system $d$ is much smaller than the distances between the cloud's center-of-mass and the detector at $\vec{R}$. The intensity of the scattered light can be calculated from the first order photon correlation function as

$$
I_{m}(\vec{R})=\left\langle a_{m}^{\dagger}(\vec{R}) a_{m}(\vec{R})\right\rangle
$$

where $a_{m}$ is a photon operator for the $m$ th spectral band with $m \in\{C, R, L\}$. If was shown in [29] that these intensities of the different spectral lines can be evaluated as

$$
\begin{aligned}
& I_{C}(\vec{R})=\frac{1}{4} \sum_{j, i=1}^{N} \Psi_{R}\left(\vec{r}_{j i}, \omega_{L}\right)\left\langle R_{z j} R_{z i}\right\rangle \sin ^{2}(2 \theta), \\
& I_{L}(\vec{R})=\sum_{j, i=1}^{N} \Psi_{R}\left(\vec{r}_{j i}, \omega_{-}\right)\left\langle R_{12}^{(j)} R_{21}^{(i)}\right\rangle \sin ^{4} \theta \\
& I_{R}(\vec{R})=\sum_{j, i=1}^{N} \Psi_{R}\left(\vec{r}_{j i}, \omega_{+}\right)\left\langle R_{21}^{(j)} R_{12}^{(i)}\right\rangle \cos ^{4} \theta
\end{aligned}
$$

Here, $\Psi_{R}\left(\vec{r}_{j i}, \omega\right)=\Psi_{R}(\omega) \exp \left[i\left(\vec{k}-\vec{k}_{L}\right) \vec{r}_{j i}\right]$ for a scattered photon of wave-vector $\vec{k}$. $\Psi_{R}(\omega)$ depends on the atom-environment coupling and in general is a function of frequency with $R=|\vec{R}| \gg k_{L}^{-1}$. As we focus on large inter-particle separations [see Eq. (1)], there is only a negligible direct coupling between the atoms, and thus the collective correlators $\left\langle R_{12}^{(i)} R_{21}^{(j)}\right\rangle$ describing the vacuummediated interactions among the emitters $i$ and $j$ decouple for $i \neq j$, that is,

$$
\left\langle R_{12}^{(i)} R_{21}^{(j)}\right\rangle \approx\left\langle R_{12}^{(i)}\right\rangle\left\langle R_{21}^{(j)}\right\rangle \quad(i \neq j) .
$$

In the strong driving field case, the atomic variable $\left\langle R_{12}^{(j)}\right\rangle$ scales as $\gamma / \tilde{\Omega}$ and therefore can be neglected in a secular approximation. As a consequence, the intensities of the spectral side-bands are proportional to the number of scatters in the ensemble, i.e., $\left\{I_{L}, I_{R}\right\} \propto N$. Near the resonance $(\theta=\pi / 4)$, the intensity of the central spectral line is also proportional to $N$. In what follows, we will restrict the analysis to the resonant driving field case in which the intensities of all spectral lines are proportional to the number of scatters $N$. In this case, the intensities of spontaneously scattered photons are distributed uniformly over the whole $4 \pi$ solid angle. Note that this is in contrast to the weak pumping case $\Omega<\gamma$, in which the first-order correlation function does show directional behavior as

$$
\begin{aligned}
I(\vec{R}) & =\Psi_{R}\left\{N\left(\frac{1}{2}+\left\langle S_{z}\right\rangle\right)\right. \\
& \left.+N(N-1)\left|\left\langle S^{+}\right\rangle\right|^{2} \cos \left[\left(\vec{k}-\vec{k}_{L}\right) \vec{r}_{j i}\right]\right\},
\end{aligned}
$$

where

$$
\begin{aligned}
\left\langle S_{z}\right\rangle & =-\frac{\gamma^{2}+\Delta^{2}}{2\left(\gamma^{2}+\Delta^{2}+\Omega^{2}\right)}, \\
\left\langle S^{+}\right\rangle & =\frac{i \Omega\left(\gamma^{2}+\Delta^{2}\right)}{(\gamma-i \Delta)\left(\gamma^{2}+\Delta^{2}+\Omega^{2}\right)} .
\end{aligned}
$$

\section{Second order correlations}

Our main observable in the following will be the the normalized second-order correlation functions $g^{(2)}(\tau)$, which is measured by two detectors positioned at $\vec{R}_{1}$ and $\vec{R}_{2}$. It is defined as

$$
g_{m n}^{(2)}\left(\tau, \vec{R}_{1}, \vec{R}_{2}\right)=\frac{G_{m n}^{(2)}\left(\tau, \vec{R}_{1}, \vec{R}_{2}\right)}{I_{m}\left(\vec{R}_{1}\right) I_{n}\left(\vec{R}_{2}\right)}
$$

i.e., as the unnormalized second order correlation function

$$
\begin{aligned}
G_{m n}^{(2)}\left(\tau, \vec{R}_{1}, \vec{R}_{2}\right)= & \left\langle a_{m}^{\dagger}\left(\vec{R}_{1}\right) a_{n}^{\dagger}\left(\tau, \vec{R}_{2}\right)\right. \\
& \left.\times a_{n}\left(\tau, \vec{R}_{2}\right) a_{m}\left(\vec{R}_{1}\right)\right\rangle,
\end{aligned}
$$

normalized to the intensities $I_{m}\left(\vec{R}_{1}\right)$ and $I_{n}\left(\vec{R}_{2}\right)$ defined in Eq. (6). The quantity $g_{m n}^{(2)}$ for $\{m, n\} \in\{C, R, L\}$ can be interpreted as a measure for the probability of detecting one photon emitted in mode $m$ and another photon emitted in mode $n$ with time-delay $\tau$. Particularly, $g^{(2)}(\tau=0)$ describes the photon statistics (e.g., sub/super-Poissonian), whereas $g^{(2)}(\tau \neq 0)$ indicates photon bunching or antibunching.

To calculate the correlation function, we assume laser driving on resonance $(\theta=\pi / 4)$, and a large atomic ensembles $(N \gg 1)$ such that the secular approximation is valid. We also assume that all possible pairs of atoms contribute equally to the second-order correlation functions. This assumption is valid as long as the angle between the wave vectors of the incident laser and the scattered photons is small, that is $\left\{\phi, \phi_{0}\right\}$ should be of order of few degrees (see Fig. 1). Finally, we for the moment consider a single interparticle distance vector $\vec{r}_{j i}$ for all pairs only, but this restriction will be relaxed later on. Based on these assumptions, we found that the correlation and cross-correlation functions of photons scattered 
into the different spectral bands can be represented as

$$
\begin{aligned}
g_{C C}^{(2)}\left(\tau, \vec{R}_{1}, \vec{R}_{2}\right) & =1+2 \cos \left(\delta_{1}\right) \cos \left(\delta_{2}\right) e^{-2 \gamma \tau}, \\
& =1+\left[\cos \left(\delta_{+}\right)+\cos \left(\delta_{-}\right)\right] e^{-2 \gamma \tau}, \\
g_{L L}^{(2)}\left(\tau, \vec{R}_{1}, \vec{R}_{2}\right) & =g_{R R}^{(2)}\left(\tau, \vec{R}_{1}, \vec{R}_{2}\right) \\
& =1+\cos \left(\delta_{-}\right) e^{-3 \gamma \tau}, \\
g_{L R}^{(2)}\left(\tau, \vec{R}_{1}, \vec{R}_{2}\right) & =g_{R L}^{(2)}\left(\tau, \vec{R}_{1}, \vec{R}_{2}\right) \\
& =1+\cos \left(\delta_{+}\right) e^{-3 \gamma \tau}, \\
g_{C X}^{(2)}\left(\tau, \vec{R}_{1}, \vec{R}_{2}\right) & =g_{X C}^{(2)}\left(\tau, \vec{R}_{1}, \vec{R}_{2}\right) \\
& =1 \quad \text { for } X \in\{L, R\} .
\end{aligned}
$$

Here,

$$
\begin{aligned}
\delta_{s} & =\left(\vec{k}_{s}-\vec{k}_{L}\right) \vec{r}_{j i}, \\
\delta_{+} & =\delta_{1}+\delta_{2}, \\
\delta_{-} & =\delta_{1}-\delta_{2},
\end{aligned}
$$

with $\vec{k}_{s}$ being the wave-vector of the photon $s$ scattered in direction $\vec{R}_{s}(s \in\{1,2\})$. Note that these results differ from the corresponding expressions for a single pair of atoms or a regular structure of atoms [29]. The reason is that for a single pair, one chooses $N=2$, whereas in deriving Eqs. (13) the assumption $N \gg 1$ was used such that terms proportional to $1 / N$ can be neglected.

\section{E. Ensemble averaging}

To estimate the signal obtained from a cloud of randomly distributed particles, the correlation functions $g_{X Y}^{(2)}\left(\tau, \vec{R}_{1}, \vec{R}_{2}\right)$ have to be averaged over the different interatomic distance vectors $\vec{r}_{i j}$ in the cloud. Since in general it can be expected that the averaging procedure affects the result, we compare three averaging procedures.

\section{Averaging over a spherical shell}

The first averaging procedure we consider has been suggested in previous works on scattering from dilute gases, see, e.g., [33]. It consists of (i) an isotropic average over the relative orientation $\mathbf{n}$ of the atoms over the unit sphere, followed by (ii) an average of the interatomic distance $r_{j i}=\left|\vec{r}_{j i}\right|$ over an interval of order of the laser wave-length, around their typical distance $l$ :

$$
\langle\cdots\rangle_{\text {conf }}=\frac{k_{L}}{(4 \pi)^{2}} \int_{l-2 \pi / k_{L}}^{l+2 \pi / k_{L}} d r_{j i} \int d \Omega_{\mathbf{n}} \cdots .
$$

It should be noted that the restriction to the radial averaging over a range of order of the wave length is not a priori justified; it is however motivated by the fact that the distance distribution of particles in a gas is peaked around a mean value, even though the distribution generally is broad. In the numerical examples below, we use $l=20 \lambda_{L}$.

\section{Averaging over a spherical volume}

This method is a modification of the first method in which we change the averaging over the radial coordinate to range from 0 to twice the volume radius $R$ :

$$
\langle\cdots\rangle_{\text {conf }}=\frac{1}{8 \pi R} \int_{0}^{2 R} d r_{j i} \int d \Omega_{\mathbf{n}} \cdots .
$$

This averaging procedure marks the opposite extreme of the averaging in Sec. IIE 1 in that a constant distance distribution over all possible distances is assumed. In the numerical examples below, we use $R=100 \lambda_{L}$.

\section{Numerical sampling}

The first two averaging methods made assumptions on the distribution of distances between the atoms, which may not be fulfilled in a gas of atoms. To investigate the effect of this further, in a third averaging procedure, we randomly place a number of atoms $N$ in a cubic volume of side length $2 R$ and then calculate the quantities in Eqs. (13) for this sample of atoms. In the numerical examples below, we use $R=100 \lambda_{L}$ and $N=300$.

\section{RESULTS}

As a first result, we note from Eqs. (13) that the second order correlation function can exceed unity in several cases together with bunching, which means that there is an enhanced probability to generate photons in pairs. For example, the second-order correlation function for the central-band photons $g_{C C}^{(2)}$ has maxima when $\vec{k}_{1}+\vec{k}_{2}=2 \vec{k}_{L}$ [see Eq. [13(a)], i.e., in forward direction. Similar results are observed for the sideband spectral lines. For example, pairs of photons can be generated in which one photon is emitted in the left spectral band, whereas the other is emitted from the right spectral sideband, see Eq.13(c). In contrast, photon pairs originating from the same sideband (left or right) are most likely for $\vec{k}_{1}=\vec{k}_{2}$ independent of the direction of the wave vector and thus do not show directional behavior, see Eq. 13.(b). Finally, there is no enhanced probability for detecting pairs of photons in which one photon is generated in the central band and one in either of the sidebands, and the corresponding correlation function does not exhibit any directionality, see Eq. 13(d).

Regarding the intensity, the unnormalized secondorder correlation functions are proportional to the number of particles in the ensemble squared, i.e. $G^{(2)}(0) \propto$ $N^{2}$.

The obtained results for the normalized second-order correlation function $g_{C C}^{(2)}(0)$ for photons emitted in the central band after the configuration average are shown in Fig. 2. It can be seen that even after the averaging, the 

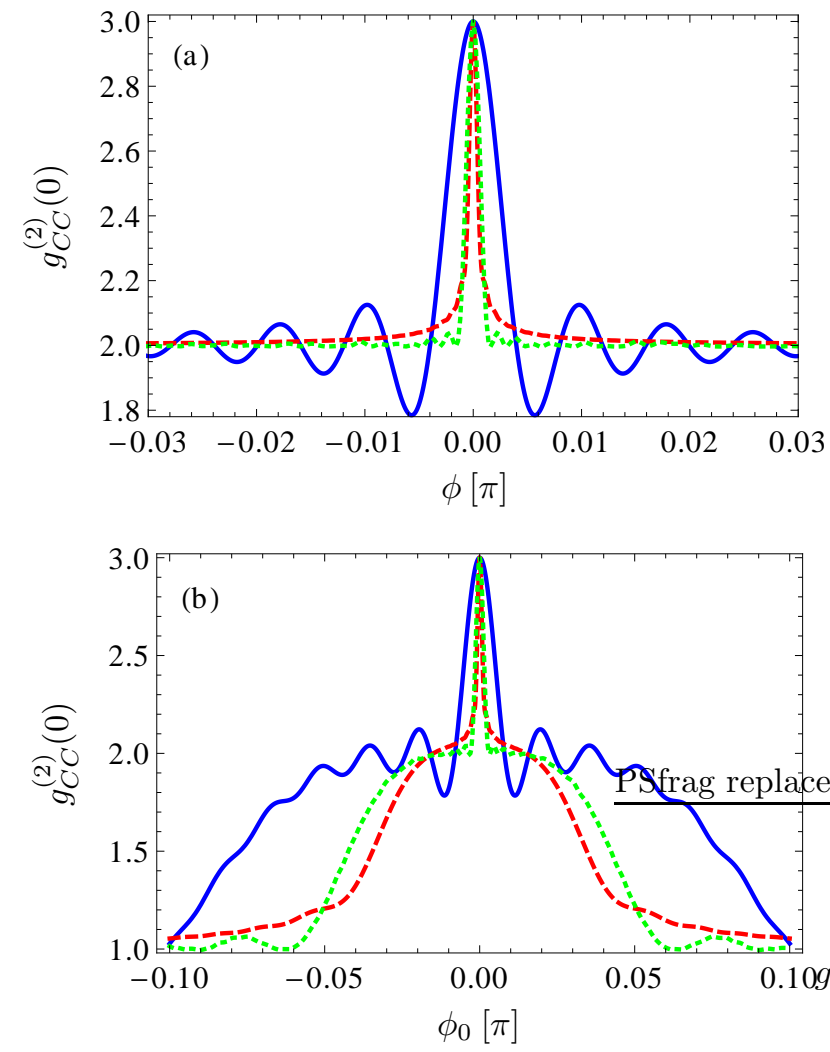

FIG. 2: (Color online) Normalized and configuration averaged second-order correlation function $g_{C C}^{(2)}(0)$ between two photons emitted from the central spectral band. In (a), the correlation function is shown for pairs of photons emitted in the same direction ( $\phi_{0}=0$, see Fig. 1), and plotted as a function of the emission direction $\phi$. In (b), the correlation function is plotted as a function of the opening angle $\phi_{0}$ between the two photons, which corresponds to the case of two distinct detectors. The two photons are measured at positions symmetric with respect to the incident laser field direction, i.e., $\phi=0$. The blue solid line shows averaging over a spherical shell, the dashed red line averaging over a spherical volume, and the green dotted line the numerical sampling.

correlation function exhibits a sharp peak around direction of the the incident laser wave-vector $\vec{k}_{L}$, indicating super-bunching. Fig. 2(a) shows the case with a single two-photon detector $\left(\phi_{0}=0\right)$ for different emission directions $\phi$. Figure 2(b) depicts the same correlation function but with two single-photon detectors placed symmetrically with respect to the laser wave-vector $\vec{k}_{L}$ direction given by $\phi=0$. Note that the correlation functions for two photons emitted both from the left or both from the right spectral sideband do not show a directionality in space.

Fig. 3 shows the corresponding results for the two photon cross correlation with one photon emitted in the left, and one in the right spectral sideband. Again, we find maxima at $\phi_{0}=0$ for two individual detectors placed symmetrically around the incident laser direction. But in contrast to the central band correlation function, this
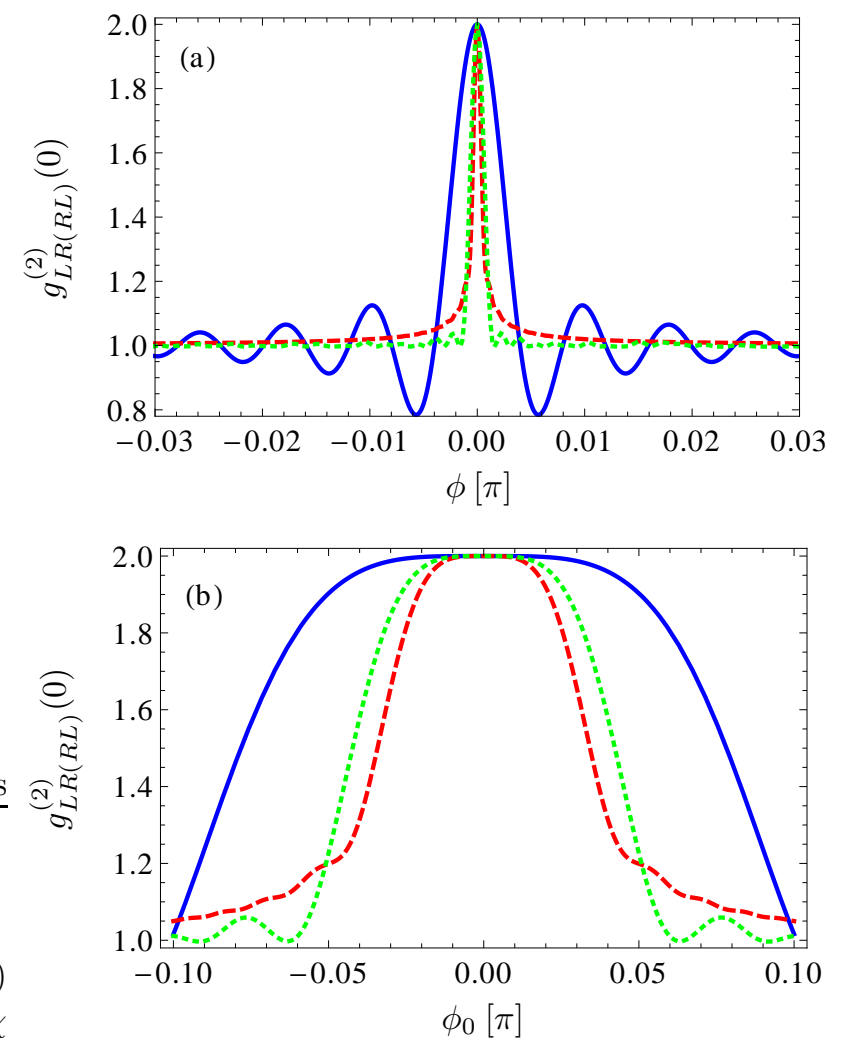

FIG. 3: (Color online) Same as Fig. 2, except that the normalized and configuration averaged second-order correlation functions $g_{L R(R L)}^{(2)}(0)$ between one photon emitted in the left and one photon emitted in the right sideband is shown.

maximum is not peaked, but rather broad. If a twophoton detector is used, a narrow maximum is observed in the forward direction, see Fig. 2(a). Interestingly, in this case, depending on the precise positioning around the forward direction and on the averaging procedure, also sub-Poissonian photon-statistics can occur.

We further define the Cauchy-Schwarz parameter

$$
\chi=\frac{g_{L L}^{(2)}(0) g_{R R}^{(2)}(0)}{\left[g_{L R}^{(2)}(0)\right]^{2}}=\frac{g_{L L}^{(2)}(0) g_{R R}^{(2)}(0)}{\left[g_{R L}^{(2)}(0)\right]^{2}},
$$

which relates the correlation between photons emitted into individual modes to the cross-correlation between photons emitted into two different modes [34]. If $\chi<1$ the Cauchy-Schwarz inequality is violated. Figure 4 shows violation of the Cauchy-Schwarz inequality for photons scattered into the side-bands.

\section{DISCUSSION AND SUMMARY}

An interpretation of the results obtained in the previous section can be found in the semiclassical dressedstate picture. Suppose one laser photon is absorbed and the atom is in the dressed-state $|\tilde{2}\rangle$. Then one option 


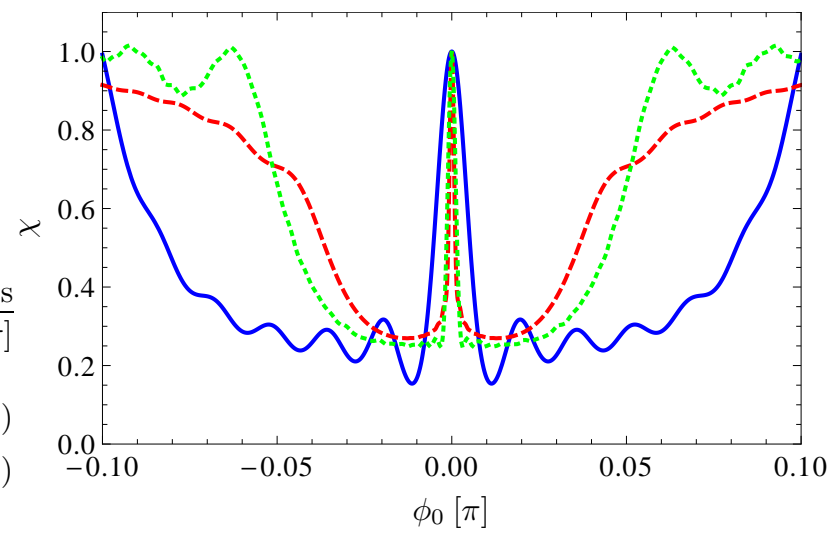

FIG. 4: (Color online) The Cauchy-Schwarz inequality $\chi$ characterizing the cross-correlations of photon pairs with one photon emitted from each sideband. The photons are detected symmetrically around the incident laser field direction $(\phi=0)$, and the result is shown as a function of the angle between the wave vectors of the two emitted photons $\phi_{0}$. The Cauchy-Schwarz inequality is violated for $\chi<1$. The curves are as in Fig. 2

will be a spontaneously emitted photon on the $|\tilde{2}\rangle \rightarrow|\tilde{2}\rangle$ transition followed by a further absorption of a laser photon during time-interval $\tilde{\Omega}^{-1}$ and subsequently decay on the same dressed-state transition. Similar effect occurs on the dressed-state transition $|\tilde{1}\rangle \leftrightarrow|\tilde{1}\rangle$ when initially the emitter is in the $|\tilde{1}\rangle$ dressed-state, and both processes contribute to the central-band scattering where $\vec{k}_{1}+\vec{k}_{2} \approx 2 \vec{k}_{L}$. Another option will be a spontaneously emitted photon on the $|\tilde{2}\rangle \rightarrow|\tilde{1}\rangle$ dressed-state transition followed by an absorption of a laser photon on the $|\tilde{1}\rangle \rightarrow|\tilde{1}\rangle$ transition and further spontaneously decay on the $|\tilde{1}\rangle \rightarrow|\tilde{2}\rangle$ dressed-state transition. Together with the reverse process where a spontaneous emission occurs on the $|\tilde{1}\rangle \rightarrow|\tilde{2}\rangle$ transition (if initially the particle is in the $|\tilde{1}\rangle$ dressed-state) followed by a laser absorption on the $|\tilde{2}\rangle \rightarrow|\tilde{2}\rangle$ transition and a subsequently decay on the $|\tilde{2}\rangle \rightarrow|\tilde{1}\rangle$ dressed-state transition, these effects describe photon correlations among the right and left side-bands, or vice versa and, again, $\vec{k}_{1}+\vec{k}_{2} \approx 2 \vec{k}_{L}$, and therefore, we have a directional-dependent photon distribution. In contrast, all other scattering processes, i.e., two-photon spontaneously emission on the same side-band or crosscorrelations involving a side-band photon and a central one do not fulfill the relation $\vec{k}_{1}+\vec{k}_{2} \approx 2 \vec{k}_{L}$, that is $\vec{k}_{1}+\vec{k}_{2} \neq 2 \vec{k}_{L}$, and thus, in these processes the photon distribution will be uniformly in space after a configuration averaging.

Comparing the results for the three averaging procedures in Figs. 2, 4, it can be seen that the averaging strategy can substantially affect the result quantitatively, even though the qualitative features of the obtained results remain similar. In particular the averaging over a spherical volume and the numerical sampling in a cubic volume of comparable size agree reasonably well. A notable difference between the three methods is the sub-Poissonian statistics found in Fig. 3(a), which is pronounced only in the case of averaging over the spherical shell. The reason is that the fringe pattern seen when plotting against $\phi$ washes out if the correlation function is averaged over a larger range of distances.

In summary, we discussed the second order correlation function for light emitted from a strongly and nearresonantly driven dilute cloud of atoms. Because of the strong driving, the fluorescence spectrum separates into distinct peaks, for which the spectral properties can be defined individually. We have shown that the secondorder correlations for various combinations of photons from different spectral lines exhibit bunching together with super- or sub-Poissonian photon statistics, tunable by the choice of the detector positions. Furthermore, we demonstrated that the Cauchy-Schwarz inequality is violated for photons emitted from the two spectral side bands. The emitted light intensity is proportional to the square of the number of particles, and thus can potentially be intense. Interesting applications might arise if the presented method is applied to generate correlated photon pairs in the x-ray domain [35 37].

\section{Acknowledgments}

The authors acknowledge valuable discussions with C. H. Keitel.
[1] R. J. Glauber, Rev. Mod. Phys. 78, 1267 (2006); L. Davidovich, ibid 68, 127 (1996); H. Paul, ibid 54, 1061 (1982).

[2] R. Hanbury Brown, R. Q. Twiss, Nature (London) 177, 27 (1956).

[3] G. Baym, Acta Phys. Pol. B 29, 1839 (1998).

[4] H. Hanbury Brown, R. Q. Twiss, Nature (London) 178, 1046 (1956).

[5] F. T. Arecchi, Phys. Rev. Lett. 15, 912 (1965); J. Beugnon, M. P. A. Jones, J. Dingjan, B. Darquie, G. Messin, A. Browaeys, P. Grangier, Nature (London) 440, 779 (2006).

[6] G. Goldhaber, S. Goldhaber, W. Lee, A. Pais, Phys. Rev.
120, 300 (1960)

[7] M. Henny, S. Oberholzer, C. Strunk, T. Heinzel, K. Ensslin, M. Holland, C. Schönenberger, Science 284, 296 (1999); W. D. Oliver, J. Kim, R. C. Liu, Y. Yamamoto, ibid 284, 299 (1999).

[8] G. Sallen, A. Tribu, T. Aichele, R. Andre, L. Besombes, C. Bougerol, M. Richard, S. Tatarenko, K. Kheng, J.-Ph. Poizat, Nature Photonics 4, 696 (2010).

[9] M. Yasuda, F. Shimizu, Phys. Rev. Lett. 77, 3090 (1996).

[10] D. N. Klyshko, Photon and Nonlinear Optics, (Gordon and Breach Science Publishers, New York, 1989).

[11] S. Du, J. Wen, M. H. Rubin, G. Y. Yin, Phys. Rev. Lett. 
98, 053601 (2007).

[12] M. Macovei, G.-x. Li, Phys. Rev. A 76, 023818 (2007).

[13] V. Balic, D. A. Braje, P. Kolchin, G. Y. Yin, S. E. Harris, Phys. Rev. Lett. 94, 183601 (2005); P. Kolchin, S. Du, C. Belthangady, G. Y. Yin, S. E. Harris, ibid 97, 113602 (2006).

[14] Ph. Grangier, G. Roger, A. Aspect, A. Heidmann, S. Reynaud, Phys. Rev. Lett. 57, 687 (1986).

[15] A. Kuzmich, W. P. Bowen, A. D. Boozer, A. Boca, C. W. Chou, L.-M. Duan, H. J. Kimble, Nature (London) 423, 731 (2003).

[16] K. Edamatsu, G. Oohata, R. Shimizu, T. Itoh, Nature (London) 431, 167 (2004).

[17] C. H. van der Wal, M. D. Eisaman, A. Andre, R. L. Walsworth, D. F. Phillips, A. S. Zibrov, M. D. Lukin, Science 301, 196 (2003).

[18] C. Wagenknecht, Che-M. Li, A. Reingruber, X.-H. Bao, A. Goebel, Yu-Ao Chen, Q. Zhang, K. Chen, J.-W. Pan, Nature Photonics 4, 549 (2010); S. Barz, G. Cronenberg, A. Zeilinger, Ph. Walther, ibid 4, 553 (2010).

[19] W. H. Peeters, J. J. D. Moerman, M. P. van Exter, Phys. Rev. Lett. 104, 173601 (2010).

[20] T. Bienaime, S. Bux, E. Lucioni, P. W. Courteille, N. Piovella, R. Kaiser, Phys. Rev. Lett. 104, 183602 (2010).

[21] I. Shani, G. Cohen, J. Fineberg, Phys. Rev. Lett. 104, 184507 (2010).

[22] S. E. Skipetrov, R. Maynard, Phys. Rev. Lett. 85, 736 (2000).

[23] R. Carminati, Phys. Rev. A 81, 053804 (2010).

[24] L. Sanchez-Palencia, M. Lewenstein, Nature Phys. 6, 87 (2010).
[25] G. Labeyrie, F. de Tomasi, J.-C. Bernard, C. A. Müller, C. Miniatura, R. Kaiser, Phys. Rev. Lett. 83, 5266 (1999); V. Shatokhin, C. A. Müller, A. Buchleitner, ibid 94, 043603 (2005).

[26] V. S. Letokhov, Sov. Phys. JETP 26, 835 (1968).

[27] M. Patra, Phys. Rev. A 65, 043809 (2002).

[28] L. S. Froufe-Perez, W. Guerin, R. Carminati, R. Kaiser, Phys. Rev. Lett. 102, 173903 (2009); W. Guerin, F. Michaud, R. Kaiser, ibid 101, 093002 (2008).

[29] M. Macovei, J. Evers, G. X. Li, C. H. Keitel, Phys. Rev. Lett. 98, 043602 (2007); M. Macovei, J. Evers, C. H. Keitel, arXiv:quant-ph/0702142 1 .

[30] B. R. Mollow, Phys. Rev. 188, 1969 (1969).

[31] P. A. Apanasevich, S. J. Kilin, J. Phys. B: At. Mol. Phys. 12, L83 (1979).

[32] C. Cohen-Tannoudji, R. Reynaud, Phil. Trans. R. Soc. Lond. A 293, 223 (1979).

[33] V. Shatokhin, C. A. Müller, A. Buchleitner, Phys. Rev. A 73, 063813 (2006).

[34] J. F. Clauser, Phys. Rev. D 9, 853 (1974); R. Loudon, Rep. Prog. Phys. 43, 58 (1980).

[35] S. W. Epp, J. R. Crespo Lopez-Urrutia, G. Brenner, V. Mäckel, P. H. Mokler, R. Treusch, M. Kuhlmann, M. V. Yurkov, J. Feldhaus, J. R. Schneider, M. Wellhöfer, M. Martins, W. Wurth, J. Ullrich, Phys. Rev. Lett. 98, 183001 (2007).

[36] Th. Bürvenich, J. Evers, and C. H. Keitel, Phys. Rev. Lett. 96, 142501 (2006).

[37] A. Pálffy, C. H. Keitel and J. Evers, Phys. Rev. Lett. 103, 017401 (2009) 